\title{
The December 2009 gamma-ray flare of 3C 454.3: the multifrequency campaign
}

\author{
L. Pacciani ${ }^{1}$, V. Vittorini ${ }^{1,2}$, M. Tavani ${ }^{1,2}$, M. T. Fiocchi ${ }^{1}$, S. Vercellone ${ }^{17}$, F. \\ D'Ammando $^{17}$, T. Sakamoto ${ }^{22,27,28}$, E. Pian ${ }^{16,23}$, C. M. Raiteri ${ }^{24}$, M. Villata ${ }^{24}$, M.

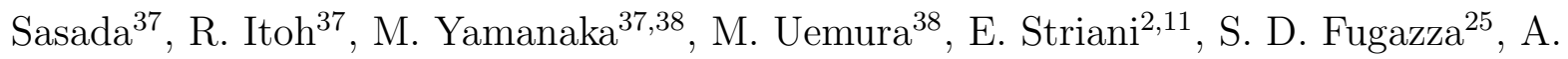 \\ Tiengo $^{3}$, H. A. Krimm ${ }^{22,28,29}$, M. C. $\mathrm{Stroh}^{30}$, A. D. Falcone ${ }^{30}$, P. A. Curran ${ }^{21}$, A. C. \\ Sadun $^{26}$, A. Lahteenmaki ${ }^{31}$, M. Tornikoski ${ }^{31}$, H. D. Aller ${ }^{32}$, M. F. Aller ${ }^{32}$, C. S. Lin ${ }^{33}$, V. \\ M. Larionov ${ }^{34,35,36}$, P. Leto $^{39}$, L. O. Takalo ${ }^{40}$, A. Berdyugin ${ }^{40}$, M. A. Gurwell ${ }^{41}$, A. \\ Bulgarelli ${ }^{5}$, A. W. Chen ${ }^{3,4}$, I. Donnarumma ${ }^{1}$, A. Giuliani ${ }^{3}$, F. Longo ${ }^{6}$, G. Pucella ${ }^{13}$, A. \\ Argan $^{1}$, G. Barbiellini ${ }^{6}$, P. Caraveo ${ }^{3}$, P. W. Cattaneo ${ }^{7}$, E. Costa ${ }^{1}$, G. De Paris ${ }^{1}$, E. Del \\ Monte $^{1}$, G. Di Cocco ${ }^{5}$, Y. Evangelista ${ }^{1}$, A. Ferrari ${ }^{18}$, M. Feroci ${ }^{1}$, M. Fiorini ${ }^{3}$, F. Fuschino ${ }^{5}$, \\ M. Galli ${ }^{8}$, F. Gianotti ${ }^{5}$, C. Labanti ${ }^{5}$, I. Lapshov ${ }^{1}$, F. Lazzarotto ${ }^{1}$, P. Lipari ${ }^{9}$, M. Marisaldi ${ }^{5}$, \\ S. Mereghetti ${ }^{3}$, E. Morelli ${ }^{5}$, E. Moretti ${ }^{6}$, A. Morselli ${ }^{11}$, A. Pellizzoni ${ }^{20}$, F. Perotti ${ }^{3}$, G. \\ Piano $^{1,2,11}$, P. Picozza ${ }^{2,11}$, M. Pilia ${ }^{12,20}$, M. Prest $^{12}$, M. Rapisarda ${ }^{13}$, A. Rappoldi ${ }^{7}$, A. \\ Rubini $^{1}$, S. Sabatini ${ }^{1}$, P. Soffitta ${ }^{1}$, M. Trifoglio ${ }^{5}$, A. Trois ${ }^{1}$, E. Vallazza ${ }^{6}$, D. Zanello ${ }^{9}$, S. \\ Colafrancesco $^{14}$, C. Pittori ${ }^{14}$, F. Verrecchia ${ }^{14}$, P. Santolamazza ${ }^{14}$, F. Lucarelli ${ }^{14}$, P. \\ Giommi ${ }^{14}$ and L. Salotti ${ }^{15}$ \\ ${ }^{1}$ INAF/IASF-Roma, I-00133 Roma, Italy \\ ${ }^{2}$ Dip. di Fisica, Univ. Tor Vergata, I-00133 Roma, Italy \\ ${ }^{3}$ INAF/IASF-Milano, I-20133 Milano, Italy \\ ${ }^{4}$ CIFS-Torino, I-10133 Torino, Italy \\ ${ }^{5}$ INAF/IASF-Bologna, I-40129 Bologna, Italy \\ ${ }^{6}$ Dip. Fisica and INFN Trieste, I-34127 Trieste, Italy \\ ${ }^{7}$ INFN-Pavia, I-27100 Pavia, Italy \\ ${ }^{8}$ ENEA-Bologna, I-40129 Bologna, Italy \\ ${ }^{9}$ INFN-Roma La Sapienza, I-00185 Roma, Italy
}


${ }^{10} \mathrm{CNR}-\mathrm{IMIP}$, Roma, Italy

${ }^{11}$ INFN Roma Tor Vergata, I-00133 Roma, Italy

${ }^{12}$ Dip. di Fisica, Univ. Dell'Insubria, Via Valleggio 11, I-22100 Como, Italy

${ }^{13}$ ENEA Frascati, I-00044 Frascati (Roma), Italy

${ }^{14}$ ASI Science Data Center, I-00044 Frascati (Roma), Italy

${ }^{15}$ Agenzia Spaziale Italiana, I-00198 Roma, Italy

${ }^{16}$ INAF-OATS, Via Tiepolo 11, I-34143 Trieste, Italy

${ }^{17}$ INAF-IASF Palermo, Via Ugo La Malfa 153, I-90146 Palermo, Italy

${ }^{18}$ Dip. Fisica, Universitá di Torino, Torino, Italy

${ }^{19}$ INAF-OAR, Monte Porzio Catone, Italy

${ }^{20}$ INAF-OAC, localita’ Poggio dei Pini, strada 54, I-09012 Capoterra, Italy

${ }^{21}$ Mullard Space Science Laboratory, University College London, Holmbury St. Mary, Dorking, RH5 6NT, UK

${ }^{22}$ Center for Research and Exploration in Space Science and Technology (CRESST), NASA

Goddard Space Flight Center, Greenbelt, MD 20771

${ }^{23}$ SNS, Piazza dei Cavalieri, 7, I-56126 Pisa, Italy

${ }^{24}$ OATO-INAF, Strada Osservatorio 20, I-10025, Pino Torinese (To), Italy

${ }^{25}$ OAB-INAF, via Brera 28, I-20121 Milano, Italy

${ }^{26}$ Univ. of Colorado, Denver U.S.A.

${ }^{27}$ Joint Center for Astrophysics, University of Maryland, Baltimore County, 1000 Hilltop Circle, Baltimore, MD 21250

${ }^{28}$ NASA Goddard Space Flight Center, Greenbelt, MD 20771

${ }^{29}$ Universities Space Research Association, 10211 Wincopin Circle, Suite 500, Columbia, MD 21044-3432

${ }^{30}$ Department of Astronomy \& Astrophysics, Pennsylvania State University, University

Park, PA 16802 
${ }^{31}$ Aalto University Metsähovi Radio Observatory, Metsahovintie 114, FIN-02540 Kylmala, Finland

${ }^{32}$ Dept. of Astronomy, University of Michigan, USA

${ }^{33}$ Institute of Astronomy, National Central University, Chung-Li, Taiwan 32054, ROC

${ }^{34}$ Astron. Inst., St.-Petersburg State Univ., Russia

${ }^{35}$ Pulkovo Observatory, St.-Petersburg, Russia

${ }^{36}$ Isaac Newton Institute of Chile, St.-Petersburg Branch

${ }^{37}$ Department of Physical Science, Hiroshima University, Kagamiyama 1-3-1, Higashi-Hiroshima 739-8526, Japan

${ }^{38}$ Hiroshima Astrophysical Science Center, Hiroshima University, Kagamiyama 1-3-1, Higashi-Hiroshima 739-8526, Japan

${ }^{39}$ INAF - Osservatorio Astrofisico di Catania, Italy

${ }^{40}$ Tuorla Observatory, Department of Physics and Astronomy, University of Turku, FI-21500 Piikkio, Finland

${ }^{41}$ Harvard-Smithsonian Center for Astrophysics, Cambridge, MA, USA

EMAIL: luigi.pacciani@iasf-roma.inaf.it

Received — 


\begin{abstract}
During the month of December, 2009 the blazar 3C 454.3 became the brightest gamma-ray source in the sky, reaching a peak flux $F \sim 2000 \times 10^{-8}$ ph $\mathrm{cm}^{-2}$ $\mathrm{s}^{-1}$ for $\mathrm{E}>100 \mathrm{MeV}$. Starting in November, 2009 intensive multifrequency campaigns monitored the 3C 454 gamma-ray outburst. Here we report the results of a 2-month campaign involving AGILE, INTEGRAL, Swift/XRT, Swift/BAT, RossiXTE for the high-energy observations, and Swift/UVOT, KANATA, GRT, REM for the near-IR/optical/UV data. The GASP/WEBT provided radio and additional optical data.

We detected a long-term active emission phase lasting $\sim 1$ month at all wavelengths: in the gamma-ray band, peak emission was reached on December 2-3, 2009. Remarkably, this gamma-ray super-flare was not accompanied by correspondingly intense emission in the optical/UV band that reached a level substantially lower than the previous observations in 2007-2008.

The lack of strong simultaneous optical brightening during the super-flare and the determination of the broad-band spectral evolution severely constrain the theoretical modelling. We find that the pre- and post-flare broad-band behavior can be explained by a one-zone model involving SSC plus external Compton emission from an accretion disk and a broad-line region. However, the spectra of the Dec. 2-3, 2009 super-flare and of the secondary peak emission on Dec. 9, 2009 cannot be satisfactorily modelled by a simple one-zone model. An additional particle component is most likely active during these states.
\end{abstract}

Subject headings: galaxies: active - galaxies: jets - galaxies: individual (3C 454.3) radiation mechanisms: non-thermal 


\section{Introduction}

The flat spectrum radio quasar 3C 454.3 (at a redshift $z=0.859$ ) turns out to be among the most active blazars emitting a broad-spectrum ranging from radio to gamma-ray energies. Blazars are a sub-class of active galactic nuclei, with the relativistic jet aligned to the line of sight. Their spectral energy distributions (SED) typically show a double humped shape, with the low energy peak lying between radio and X-rays, and the high energy peak in the GeV-TeV band Urry and Padovani (1995). Detailed description of blazar leptonic emission models can be found in Maraschi et al. (1992); Marsher \& Bloom (1992); Sikora et al. (1994). The observed spectra can also be modelled in the framework of hadronic models Mucke and Protheroe (2001); Mucke et al. (2003); Bottcher (2007).

Starting in 2004-2005, 3C 454.3 showed a long period of optical activity, with variability timescale ranging from several months to less than one day. In May 2005, the source reached a peak magnitude $R \simeq 12$ showing strong 1-day variability Villata et al. (2006). A radio peak was detected 9 months after the optical peak, with a flux of 22 Jy at 37 $\mathrm{GHz}$, and $20 \mathrm{Jy}$ at $43 \mathrm{GHz}$ Villata et al. (2007). The source was then quiescent from the beginning of 2006 until mid-2007 (with an R magnitude between 15 and 16, Raiteri et al. 2008). Starting in the second half of 2007 Vercellone et al. (2008), hyas been detected in a high gamma-ray state by AGILE Tavani et al. (2009), and, subsequently also by Fermi-LAT Atwood et al. (2009); Abdo et al. (2009). Typically, the level of gamma-ray activity (with a flux of $300-600 \times 10^{-8} \mathrm{ph} \mathrm{cm}^{-2} \mathrm{~s}^{-1}$ for $\mathrm{E}>100 \mathrm{MeV}$ ) has been observed to be correlated with the optical emission. Relatively large gamma-ray fluxes were detected during the AGILE observations of 2007 Vercellone et al. (2008, 2009); Donnarumma et al. (2009). During 2008, the source that was bright at the beginning of the year, started to fade in optical band Villata et al. (2009). AGILE detected the fading in gamma-rays too Vercellone et al. (2010). Fermi reported an averaged spectrum above $200 \mathrm{MeV}$ with a 
photon index $\alpha \simeq 2.3$ and a spectral break at $E_{c} \sim 2.4 \mathrm{GeV}$, obtained in August, 2008 Abdo et al. (2009).

\section{The multifrequency campaign}

The intensive monitoring of çarried out by our group covered the period of extraordinary gamma-ray activity in November-December, 2009 Striani et al. (2009). The campaign involved AGILE for the gamma-ray band, Swift/BAT, RossiXTE/HEXTE and INTEGRAL/IBIS in the hard X-ray band, RossiXTE/PCA and Swift/XRT in X-rays, Swift/UVOT in the optical and UV bands, the KANATA observatory and GRT in the optical, REM in the near infrared and optical. AGILE observed the source every day in spinning mode, scanning about $70 \%$ of the whole sky every 6 minutes. INTEGRAL pointed the source in response to a Target of Opportunity observations (ToO) Vercellone et al. (2009b), and observed it from 2009 December 6 until 2009 December 12. The RossiXTE satellite observed 3C 454.3 on 2009 December 5 and then daily from December 8 until December 17, 2009 for typical integrations of $\sim 3$ ks. Swift started to observe on 2009 November 27 , in response to a $\mathrm{ToO}$, and pointed at the source every day (UVOT performed most of the observations with the UV filters). The KANATA $1.5 \mathrm{~m}$ telescope performed a long source monitoring in the V-band, with a time-step of 1 day. The fully automated 14" GRT (Goddard Robotic Telescope) performed observations in the V and R bands, quasi-simultaneously with Swift, starting on 2009 November 30. REM started the monitoring on 2009 December 10 in response to a ToO, and observed the source every day in the VRIJHK filters. The GLAST-AGILE Support Program (GASP Villata et al. 2008, 2009) performed an intensive monitoring-campaign of the source in 2009-2010. We used a sub-sample of their data: Optical observations reported in this paper were performed at: Lulin, New Mexico Skies, Roque de los Muchachos (KVA), and St. Petersburg. GASP 
radio data were taken at Mauna Kea (SMA, $230 \mathrm{GHz}$ ), Noto (43 GHz), Metsähovi (37 $\mathrm{GHz})$, and UMRAO $(4.8,8.0$, and $14.5 \mathrm{GHz})$.

\section{Data analysis}

AGILE/GRID data were analyzed using the Build-19 software and the response matrix v10 calibrated in the energy range 100-3000 MeV. Well reconstructed gamma-ray events were selected using the FM3.119 filter. All the events collected during the passage in the South-Atlantic Anomaly were rejected. We filtered out the Earth-albedo, rejecting photons coming from a circular region of radius $85 \mathrm{deg}$ and centered on the Earth. We rejected photons coming from outside the 35 degrees from the optical axis. Gamma-ray data were analyzed with integrations of 1 or 2 days, depending on the source flux. We used the standard AGILE Maximum-Likelihood procedure (ALIKE) (see Mattox et al. 1996, for the concept definition) for each data set. The integration over 5 weeks from 2009-11-18 to 2009-12-23 UTC yields a photon index $1.88 \pm 0.08$ (all the errors reported in the paper are at $1 \sigma$, except where stated).

The INTEGRAL-IBIS Ubertini et al. (2003) data were processed using the OSA software version 8.0. light curves (from 20 to $200 \mathrm{keV}$ ) and spectra (from 18 to $200 \mathrm{keV}$ ) were extracted for each individual science window of revolutions 873 and 874 .

The Swift-BAT survey data were obtained applying the "BAT FOV" option. The data have been processed by batsurvey script available through HEASOFT software package with a snapshot (single pointing) interval. To estimate the background, ten background points around the source in a radius of $50^{\prime}$ are selected. The source, ten background points

and the bright hard X-ray sources (for cleaning purpose) are included in the input catalog of batsurvey. The BAT count rate in the $14-195 \mathrm{keV}$ band has been converted into the energy 
flux assuming a power-law photon index 1.7 as determined from the INTEGRAL-ISGRI data, see below). In order to match with the HEXTE range, the BAT hard X-ray flux has been rescaled in Fig. 1 to the $20-40 \mathrm{keV}$ band.

RossiXTE-PCA Jahoda et al. (1996) and HEXTE Rothschild et al. (1998) data were analyzed following the same procedure described in Vercellone et al. (2010). The data analysis was restricted to the PCU2 in the $3-20 \mathrm{keV}$ energy range for the PCA and to the Cluster B in the 18-50 keV range for the HEXTE. The net exposure times were 27.3 ks for PCA and $7.3 \mathrm{ks}$ for HEXTE. The background subtracted source spectra obtained with both the instruments were simultaneously fit 1 with an absorbed power-law model, with the photoelectric absorption fixed to $0.134 \times 10^{22} \mathrm{~cm}^{-2}$ Villata et al. (2006). After the introduction of a $2 \%$ systematic error, the best-fit value for the photon index is $1.74 \pm 0.01$.

The Swift-XRT data were processed using the most recent calibration files available. We utilized Swift Software version 3.5, FTOOLS version 6.8, and XSPEC version 12.5.1n. We fitted the data with an absorbed power-law model. We obtained photon indices between $1.51 \pm 0.09$ and $1.73 \pm 0.11$, and excess absorption between $(0.09 \pm 0.06) \times 10^{22}$ and $(0.17$ $\pm 0.03) \times 10^{22} \mathrm{~cm}^{-2}$ (all uncertainties on xrt spectral fit are at $90 \%$ level)

Swift-UVOT data from each observation sequence were processed by the standard UVOT tool uvotsource using the same version of the Swift software as for the XRT analysis. An extraction region of radius 5 arcsec centered on the source and a suitable background region were used. Magnitudes are based on the UVOT photometric system Poole (2008).

\footnotetext{
${ }^{1}$ The resulting fit is not statistically acceptable $\left(\chi_{\text {red }}^{2}=1.53 / 54\right.$ d.o.f. $)$; however, the addition of a $2 \%$ systematic error to the data, which is well within the expected uncertainties in the spectral calibration, is sufficient to make the fit fully acceptable $\left(\chi_{\text {red }}^{2}=1.02 / 54\right.$ d.o.f. $)$.
} 
The optical photometry of the Kanata Observatory data was performed using TRISPEC Watanabe et al. (2005). The observations were pipeline-reduced, including bias removal and flat-field corrections. We derived the $V$-band magnitude from differential photometry with a nearby reference star, USNOB 1061-0614254 ( see Gonzales-Perez et al. 2001). Photometric stability of this star has been confirmed by our simultaneous photometry for another nearby star, USNOB 1061-0614207.

All REM raw optical and NIR frames, obtained with ROSS Tosti et al. (2004) and REMIR Conconi et al. (2004) respectively, as well as images from GRT were corrected for dark, bias, and flat field following standard recipes. Instrumental magnitudes were obtained via aperture photometry, and absolute calibration has been performed by means of secondary standard stars in the field Raiteri et al. (1998).

Even if it is not reported in Figure 1, the Metsähovi radio data at $37 \mathrm{GHz}$ show a high flux with an increasing trend from 2009 December 1 until 2010 January 14, and a mean flux of $\sim 20 \mathrm{Jy}$ during the first week of December, 2009. The mean $230 \mathrm{GHz}$ flux is $\sim 25 \mathrm{Jy}$. These radio fluxes 2 are comparable to the peak flux measured in 2006 Villata et al. (2007).

All UV/optical/NIR data presented here were corrected for the Galactic extinction toward 3C 454.3 assuming $A_{V}=0.349$ Schlegel et al. (1998).

\section{Results}

The multifrequency light curves of a̧re reported in Figure 1, The exceptional gammaray flaring activity is produced during an extended period lasting several weeks. The

\footnotetext{
${ }^{2}$ The long term radio and optical light curves of ḑuring the 2009-2010 observing season will be presented in a forthcoming paper (Raiteri et al., in preparation).
} 


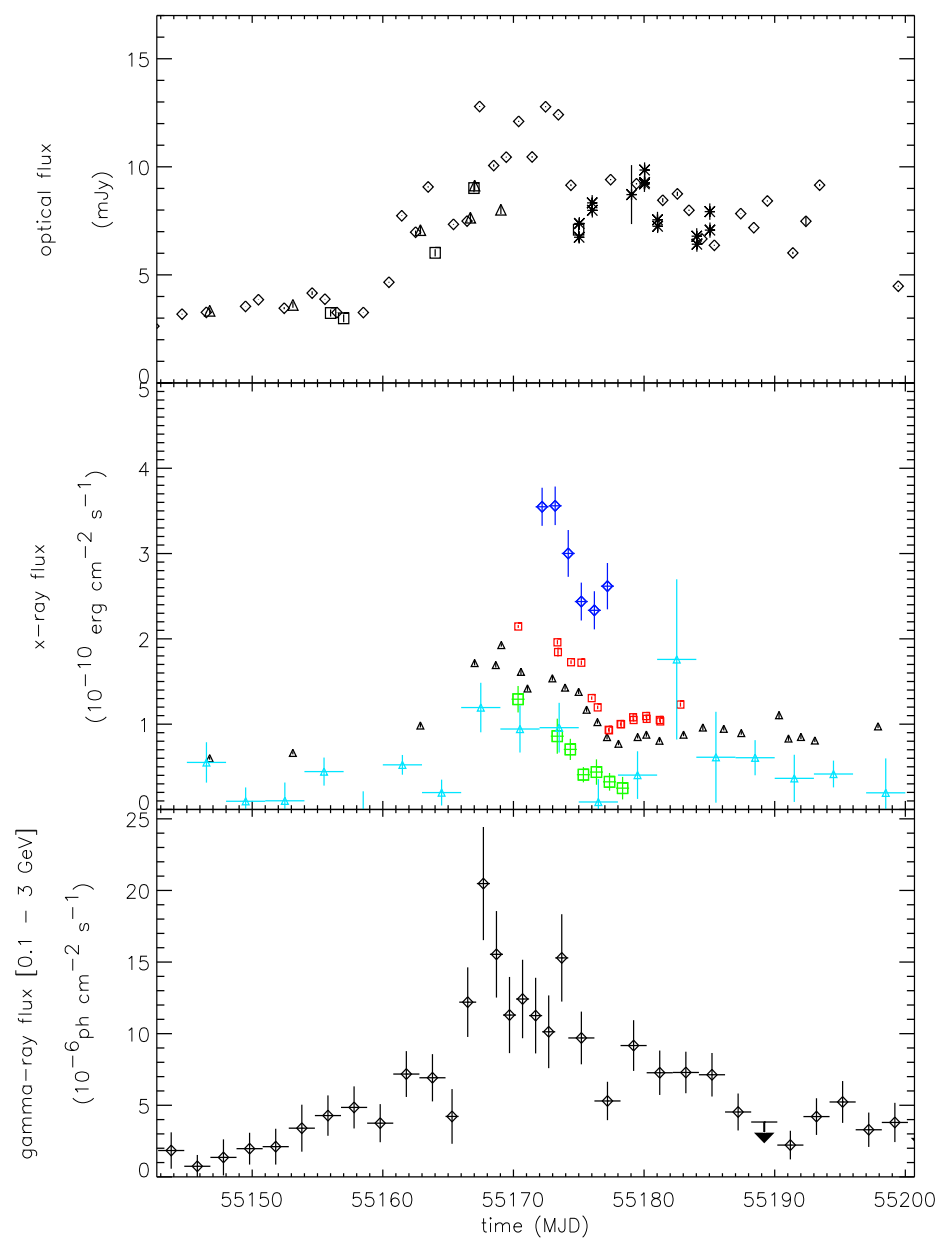

Fig. 1.- Multifrequency data collected during the campaign (Upper panel:) Optical V data from: KANATA (diamonds), REM (star), GRT (squares), UVOT (triangles). (Middle panel:) X-ray data from XRT (0.3-10 keV flux, black triangles), PCA (3-20 keV, red squares), HEXTE (20-40 keV, green squares), BAT (14-195 keV rate, rescaled to the 20-40 keV band, cyan triangles), ISGRI (20-200 keV, blue diamonds). (Bottom panel:) gamma-ray data from the AGILE-GRID (0.1-3 GeV, black diamonds). 
broad-band coverage is excellent, and for the first time allows a detailed multi-wavelength study of a gamma-ray blazar peaking above the Vela pulsar flux (for comparison, see the multifrequency coverage for the gamma-ray super-flare of PKS 1622-29, Mattox et al. 1997).

The optical data show variability timescale as short as one day or less. Due to the typical interval between optical observations (1 day), we did not show in Fig. 1 a detailed representation of the variability. The optical $\mathrm{V}$ data indicate a low-state of the source (V $>15$ mag, flux < 3.9 mJy) until MJD 55160, after which the flux started to increase. The optical flux increased of about 50\% in less then one day from MJD 55166.4 until MJD 55167.4 , reaching the value $V=13.7 \mathrm{mag}$ (e.g., $12.8 \mathrm{mJy}$ ), followed by a fast flux decrease to $V=14.3 \mathrm{mag}$ (e.g., $7.4 \mathrm{mJy}$ ) at MJD 55169.0. Another optical peak was reached at MJD 55172.5 (V=13.7 mag), and then was followed by a minimum at MJD 55175.0, with $\mathrm{V}=14.4 \mathrm{mag}$ (e.g., $6.7 \mathrm{mJy})$.

In general, the X-ray flux follow 3 the rising part of the optical emission in the interval MJD 55150-55169. Starting on MJD 55169, the X-ray flux started to fade, with the optical emission remaining in a relatively high state for 4-5 days. Our INTEGRAL hard X-ray data sample the fading phase of the high-activity period.

We performed a Discrete Correlation Function (DCF) analysis for the optical-gamma, and soft x-gamma data set. No significant delay is found within 2 days (compatible with the average bin of gamma-ray light curve).

We focused on the time-dependent spectral analysis of this exceptional activity of 3C 454.3 , and used simultaneous broad-band data to obtain a detailed account of the source variability. We obtained the SED for four periods. The first period (interval-1) is for a

\footnotetext{
${ }^{3}$ We note that no X-ray data were obtained in exact correspondence with the gamma-ray super-flare of Dec. 2-3, 2009.
} 

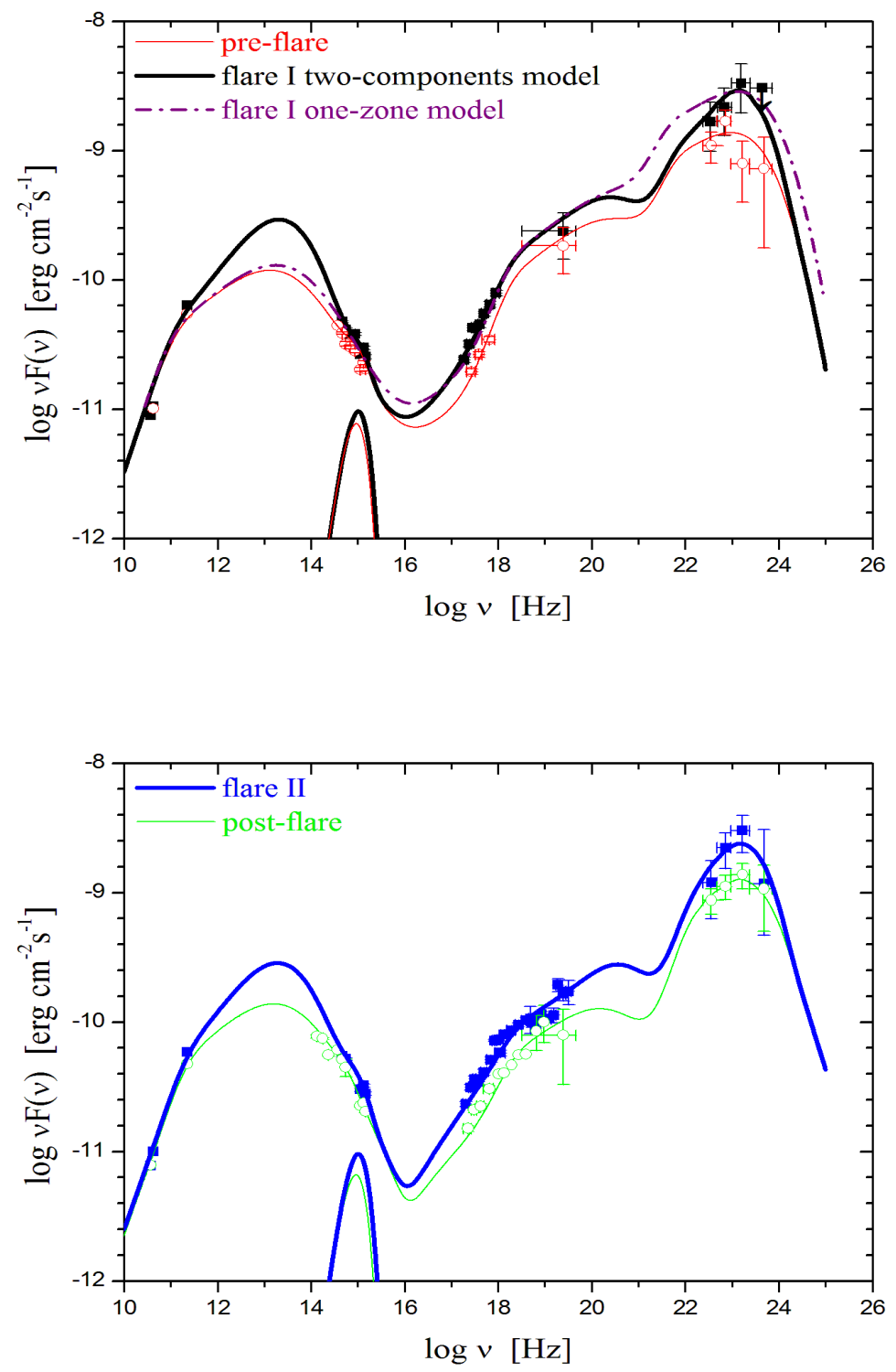

Fig. 2.- Multifrequency simultaneous spectra of ąt four different epochs superimposed with spectral modelling. Top panel: pre-flare (red open circles) and super-flare (black filled squares). Bottom panel: secondary flare (blue filled squares) and post-flare spectra (green open circles). Models details are explained in the text. 
5-day integration of GRID data, centered on 2009-11-27 09:36 UT (MJD 55162.4), and using the Swift observation at MJD 55162.9, and quasi-simultaneous GASP optical data (from KVA and New Mexico Skies) obtained within 16 hours from the Swift observation (pre-flare SED).

The second period (interval-2) was obtained for the gamma-ray super-flare episode integrating the GRID data for 1 day centered at 2009-12-02 16:48 UT (MJD 55167.7), and using of the simultaneous Swift and GRT observations at MJD 55167.0 (first flare SED). The Kanata observatory measured a flux 40\% higher in V band 10 hours after the GRT observation.

The third SED was obtained (interval-3) integrating GRID data for 2 days centered at 2009-12-06 16:48 UT (MJD 55172.7), to match the local maximum of the optical light curve with $\mathrm{V}=13.7 \mathrm{mag}$, that is apparently coincident with the secondary gamma-ray maximum near MJD 55174. For this interval we used the INTEGRAL-ISGRI data collected between MJD 55171.7 and MJD 55174.2, Swift data at MJD 55173.9, RossiXTE data at 55173.4, and GASP optical data (from Lulin and GRT) obtained within 26 hours from the Swift data.

The last SED (interval-4) was obtained integrating GRID data for 5.5 days centered at 2009-12-15 18:00 UT (MJD 55180.8), and making use of the Swift observation at MJD 55179.1, the RossiXTE observation at MJD 55179.2, the GASP optical data (from St. Petersburg and Lulin) simultaneous with Swift within 12 hours, and the near-infrared observations from REM at MJD 55181.0 (post flare SED).

Radio data for the SEDs were taken by the GASP, using observations from Mauna Kea (SMA, $230 \mathrm{GHz}$ ), Noto (43 GHz), Metsähovi (37 GHz) UMRAO (4.8, 8.0, $14.5 \mathrm{GHz}$ ), simultaneous within a few days with the XRT observations. Due to the slow variability of the radio data, we use interpolated radio values in the SEDs. The results are shown in Fig. 2, 


\section{Discussion}

The multifrequency data of the extensive campaign on şhow a remarkable behavior of the source. Starting from MJD 55150, first an overall rise of the gamma-ray emission and then of the X-ray and optical fluxes is detected. This rise culminates with peak optical/X-ray/gamma-ray emission during a 10-day period centered around MJD 55173. Subsequently, the overall flux decreased and reached again a relative-minimum state around MJD 55200. During the 2-month period the optical and X-ray fluxes vary within a factor of 3 , whereas the $\gamma$-ray flux grows by a factor of 5-10 compared to the pre-flare value. During the rapid super-flare around MJD 55167.7 the $\gamma$-ray flux doubles within 1 day with the optical and average X-ray increase of $50 \%$ and 30\%, respectively.

We find an overall correlation at all wavelengths for both long and short timescales. However, the unusual gamma-ray flaring and super-flaring activity from ḑuring the period November-December, 2009 is not accompanied by strong emission of similar intensity in the optical or even in the soft X-ray bands. The hard x-ray flux is comparable to the level of spring 2005, e.g., $\sim 3.5 \times 10^{-10} \mathrm{erg}^{2} \mathrm{~cm}^{-1}$ in $20-200 \mathrm{keV}$ Pian et al. (2006). This flaring behavior appears to be quite different from other episodes detected in 2007 and 2008 (e.g. Vercellone et al. 2009; Donnarumma et al. 2009). The synchrotron emission appears to be quite broad and centered around $\nu \sim 10^{13} \mathrm{~Hz}$.

Striani et al. (2010) provided a first report of the AGILE-GRID data. A single power-law approximation gives a photon spectral index $1.66 \pm 0.32$ in the energy band 0.1-1 GeV, integrating the data for two days centered at MJD 55167.7. We confirm this result in the analysis presented here integrating gamma-ray data for 1 day, as shown in 
Table 1: Models parameters used for producing the spectral analysis of Fig. 2. The columns give the emission interval specification, the leptonic component that dominates the gamma-ray emission, the magnetic field $B$, the comoving emission radius $R$, the particle energy distribution normalization $K$, the break energy (Lorentz factor) $\gamma_{b}$, the minimum particle energy $\gamma_{\min }$, and the low- $\left(\zeta_{1}\right)$ and high-energy spectral indices $\left(\zeta_{2}\right)$ defined as $N(\gamma)=K \gamma_{b}^{-1} /\left[\left(\gamma / \gamma_{b}\right)^{\zeta_{1}}+\left(\gamma / \gamma_{b}\right)^{\zeta_{2}}\right]$. The relativistic Maxwellian is defined as $N(\gamma)=K\left(\gamma / \gamma_{b}\right) \exp \left(-\gamma / \gamma_{b}\right)$

\begin{tabular}{|c|c|c|c|c|c|c|c|c|c|c|}
\hline Interval & Model & Component & $\begin{array}{c}\text { B } \\
(\mathrm{G})\end{array}$ & $\begin{array}{c}\mathbf{R} \\
(\mathrm{cm})\end{array}$ & $\begin{array}{c}\mathbf{K} \\
\left(\mathrm{cm}^{-3}\right)\end{array}$ & $\gamma_{\mathbf{b}}$ & $\gamma_{\min }$ & $\zeta_{1}$ & $\zeta_{2}$ & Comments \\
\hline \multirow[t]{2}{*}{1 (pre-flare) } & \multirow[t]{2}{*}{ two-comp. } & component-1* & 0.6 & $7 \times 10^{16}$ & 2.2 & 800 & 35 & 2.35 & 4.5 & broken PL \\
\hline & & component-2 & - & - & - & - & - & - & - & - \\
\hline \multirow[t]{3}{*}{2 (super-flare) } & \multirow[t]{2}{*}{ two-comp. } & component-1 & 0.6 & $7 \times 10^{16}$ & 2.2 & 800 & 35 & 2.35 & 4.5 & broken PL \\
\hline & & component-2 & 0.9 & $3 \times 10^{16}$ & 180 & 180 & 1 & - & - & relativistic Maxwellian \\
\hline & \multicolumn{2}{|c|}{ one-zone } & 0.5 & $7 \times 10^{16}$ & 2.5 & 1000 & 35 & 2.35 & 4.5 & broken PL \\
\hline \multirow[t]{2}{*}{3 (secondary-flare) } & \multirow[t]{2}{*}{ two-comp. } & component-1 & 0.6 & $7 \times 10^{16}$ & 2.5 & 800 & 45 & 2.25 & 4.5 & broken PL \\
\hline & & component-2 & 0.9 & $3 \times 10^{16}$ & 170 & 170 & 1 & - & - & relativistic Maxwellian \\
\hline \multirow[t]{2}{*}{4 (post-flare) } & \multirow[t]{2}{*}{ two-comp. } & component- $1^{*}$ & 0.6 & $7 \times 10^{16}$ & 2.5 & 800 & 45 & 2.25 & 4.5 & broken PL \\
\hline & & component-2 & - & - & - & - & - & - & - & - \\
\hline
\end{tabular}

$\left({ }^{*}\right)$ For the pre- and post-flare intervals this set of parameters describes also the simple onezone model. 
our Fig. 2 (left panel). In addition, we found a similar spectral shape of the gamma-ray emission during the secondary maximum of interval-3. Our Fig. 2 (right panel) shows the interval-3 SED as compared with the post-flare SED of interval-4.

The simultaneous observations of by AGILE, INTEGRAL, RossiXTE and Swift strongly constrain the emission models in the high-energy range. In particular, our interval-3 spectrum (blue solid squares of Fig. 2, right panel) represents one of the best constrained multifrequency spectra ever obtained for a flaring blazar from X-ray up to GeV energies.

We present in Fig. 2 the results of our spectral modelling based on Synchrotron-Self Compton (SSC), plus contribution from external seed photons (EC). We used parameters similar to those already implemented to model previous gamma-ray flares of 3C 454.3. Vercellone et al. (2009); Donnarumma et al. (2009). We find that the pre- and post-flare spectra (interval-1 and interval-4) are adequately represented by a simple one-zone SSC model plus EC in which the accretion disk and the broad-line region provide the necessary soft radiation field for the inverse Compton components that dominate the X-ray through the GeV energies. The simple one-zone model parameters for the pre- and post-flare intervals are reported in Table 1 (they are also labelled component-1 in the table).

We can fit the super-flare with a simple one-zone model as shown in Figure 2 (dot-dashed curve), with the parameters reported in Table 1. Such a model requires (compared to the pre-flare fit parameters) an increase of the electron energy and density, and a slight reduction of the comoving magnetic field for the whole electron population of the blob (as also reported in Bonnoli et al. 2010). We note, however, that we used the optical data obtained during the rising edge of optical emission for the SED of the super-flare. Using only the $\mathrm{V}$ peak emission for the optical portion of the spectra, the one-zone model gives a magnetic field of $0.55 \mathrm{G}$, closer to the value for the pre-flare. 
The time evolution of the source (e.g., the lack of strong optical emission and the gamma-ray spectrum during the super-flare) can be also described adopting a different approach. We assume a long-term rise and fall of the mass accretion rate onto the central black hole. This enhanced accretion causes an overall increase of the synchrotron emission and of the soft photon background scattered off by the primary component of accelerated electrons (component-1). An additional population of accelerated leptons (component-2, co-existing with component-1) can be introduced for the super-flare and secondary flare episodes. This component is a consequence of additional particle acceleration and/or plasmoid ejection near the jet basis. Table 1 reports the parameters that we used to model the Fig. 2 spectra (the two-component models are reported as thick solid lines for the super-flare and for the secondary flare). For all intervals, we assume the presence of component-1. This is the only component acting for the pre- and post-flare intervals, with the parameters given in Table 1. The super-flare (secondary-flare) parameters for the component-1 are the same as the pre-flare (post-flare) interval. We assumed a bulk Lorentz factor $\Gamma=25$, a jet angle with respect with the line of sight $\theta=1.2^{\circ}$, an accretion disk of bolometric luminosity $L_{d}=6 \times 10^{46} \mathrm{erg} \mathrm{s}^{-1}$ slowly decaying toward $L_{d}=5 \times 10^{46} \mathrm{erg} \mathrm{s}^{-1}$. A broad line region located 0.5 pc from the black hole reflects $5 \%$ of the disk power toward the emitting regions.

The component-2 energy distribution that better reproduces our gamma-ray spectral data is a relativistic Maxwellian of characteristic energy $\gamma_{b} \simeq 180$. Interestingly, this component appears to be strongly energized but not yet modified by additional non-thermal acceleration.

This two-component approach avoids the problem of explaining the time variability of the physical parameters of the whole electron population of the one-zone model, at the price of adding an additional varying component superimposed with the pre-existing one. 
To summarize, our multifrequency data for the December, 2009 flare of provide a wealth of very important information on this puzzling and fascinating blazar. We find that is characterized by strong broad-band spectral variability, and that the modelling of the peak gamma-ray emission episodes suggests more elaborate models than the standard one-zone SSC+EC models of bright blazars.

The AGILE Mission is funded by the Italian Space Agency (ASI) with scientific and programmatic participation by the Italian Institute of Astrophysics (INAF), and the Italian Institute of Nuclear Physics (INFN). This investigation was carried out with partial support from the ASI contract n. I/089/06/2. V.Larionov acknowledges support from Russian RFBR foundation via grant 09-02-00092. The operation of UMRAO is made possible by funding from the NSF, NASA, and the University of Michigan. The Submillimeter Array is funded by the Smithsonian Institution and the Academia Sinica Institute of Astronomy and Astrophysics. The GASP president acknowledges the ASI support through contract ASI-INAF I/088/06/0. 


\section{REFERENCES}

Abdo, A. A., 2009, ApJ, 699, 817-823

Atwood et al, 2009, ApJ, 697, 1071-1102

Bonnoli, G. et al, 2010, MNRAS submitted, arXiv:1003.3476

Bottcher, M., 2007, Ap\&SS, 309, 95-104

Conconi, P., 2004, SPIE, 5492, 1602-1612

Donnarumma, I. et al, 2009, ApJ, 707, 1115-1123

Gonzalez-Perez et al. 2001, AJ, 122, 2055

Jahoda, K, et al, 1996, SPIE, 2808, 59-70

Maraschi. L., Ghisellini, G., and Celotti, A., 1992, ApJ, 397, L5

Marscher, A. P., and Bloom, S. D., 1992, Proceedings of The Compton Observatory Science Workshop, 346

Mattox, J. R., Bertsch, D. L., Chiang, J., et al., 1996, ApJ, 461, 396

Mattox, J. R., Wagner, S. J., Malkan, M. et al., 1997, ApJ, 476, 692

Mucke, A., Protheroe, R. J., 2001, Astropart. Phys, 15, 121-136

Mucke, A. et al, 2003, Astropart. Phys, 18, 593-613

Urry, C. M., and Padovani, P., 1995, PASP, 107, 803

Pian, E. et al, 2006, A\&A, 449, L21-L25

Poole, T. S., Breeveld, A. A., Page, M. J., et al., 2008, MNRAS, 383, 627 
Raiteri, C.M. et al. 1998, A\&A, 130, 495

Raiteri, C.M. et al. 2008, A\&A, 491, 755-766

Rothschild, R. E. et al, 1998, ApJ, 496, 538

Schlegel, Finkbeiner, \& Davis 1998, ApJ, 500, 525

Sikora, M., Begelman M. C., and Rees, M., 1994, ApJ, 421, 153

Striani, E et al., 2009, ATel 2322; ATel 2326

Striani, E et al., 2010, ApJ, submitted

Tavani, M., Barbiellini, G., Argan, A., et al., 2009, A\&A, 502, 995-1013

Tosti, G. et al, 2004, SPIE, 5492, 689-700

Ubertini, P. et al, 2003, A\&A, 411, L131-L139

Vercellone, S. et al, 2008, ApJ, 676, L13-L16

Vercellone, S. et al, 2009, ApJ, 690, 1018-1030

Vercellone, S. et al, 2009, ATel 2344

Vercellone, S. et al, 2010, ApJ, 712, 405

Villata, M. et al, 2006, A\&A, 453, 817-822

Villata, M. et al, 2007, A\&A, 464, L5-L9

Villata et al, 2008, A\&A, 481, L79-L82

Villata et al, 2009, A\&A, 504, L9-L12

Watanabe, M., et al. 2005, PASP, 117, 870-884 
Zerbi, R. M., 2001, AN, 322, 275-285.

This manuscript was prepared with the AAS LTEX macros v5.2. 